\title{
Zonisamide in the management of epilepsy in the elderly
}

\author{
This article was published in the following Dove Press journal: \\ Clinical Interventions in Aging \\ 8 June 2015 \\ Number of times this article has been viewed
}

\author{
Andrea Romigi ${ }^{1,2}$ \\ Eti A Femia ${ }^{3}$ \\ Cinzia Fattore ${ }^{4}$ \\ Giuseppe Vitrani' \\ Giancarlo Di Gennaro' \\ Valentina Franco ${ }^{4}$ \\ 'Istituto Neurologico Mediterraneo, \\ IRCCS Neuromed, Pozzilli (IS), Italy; \\ ${ }^{2}$ Neurophysiopathology Department, \\ University of Rome Tor Vergata, \\ Rome, Italy; ${ }^{3}$ Dipartimento di Scienze \\ della Salute, Università degli Studi \\ di Milano, Milano, Italy; ${ }^{4}$ Clinical \\ Trial Centre \& Antiepileptic Drugs, \\ C. Mondino National Neurological \\ Institute, Pavia, Italy
}

\begin{abstract}
Zonisamide (ZNS), a second-generation antiepileptic drug, indicated as add-on treatment of focal epilepsy, has been recently approved as monotherapy for the treatment of partial seizures in adults affected by newly diagnosed epilepsy in Europe. Evidence on the efficacy and tolerability of antiepileptic drugs in the elderly is still lacking as these patients are frequently excluded from clinical trials. Here, a comprehensive overview of available data regarding the use of ZNS in the treatment of epilepsy in elderly people is provided. In a pooled analysis conducted in patients aged $\geq 65$ years, no new/unexpected safety findings have emerged. Few data from uncontrolled investigations suggest that ZNS may be effective and well tolerated when administered as monotherapy or adjunctive antiepileptic treatment in the elderly. However, evidence from these observational studies is less than satisfactory, and randomized controlled trials focused on these patients are still needed.
\end{abstract}

Keywords: zonisamide, elderly, epilepsy, safety, efficacy

\section{Introduction}

Epilepsy is one of the most serious disorders of the brain with about 50 million people affected worldwide. ${ }^{1}$ The incidence of epilepsy is highest at the younger and older age, and around $30 \%$ of new epilepsies arise in older patients. The prevalence rate of epilepsy in subjects aged over 70 years is quite twice that in children, ${ }^{2}$ and the incidence rates increase significantly with age for every decade after 60 years of age., ${ }^{1,3}$ With life expectancy becoming progressively longer, especially in high-income countries, the prevalence of epilepsy in the elderly should increase and to understand its epidemiology is a matter of significant public health importance. Evidence on the optimal treatment of epilepsy in old age is limited, mainly because older patients are often excluded from randomized clinical trials. ${ }^{4}$ Because of the unfavorable pharmacokinetic and safety profile ${ }^{5,6}$ of first-generation antiepileptic drugs (AEDs) in the elderly, there has been an increasing attention on newer pharmacological alternatives for this population. However, comparative randomized double-blind studies about the use of second-generation AEDs in older subjects with newly diagnosed epilepsy are few, ${ }^{7-9}$ and only observational studies have been carried out for some medications. ${ }^{10-12}$

Zonisamide (ZNS), a second-generation AED, has been recently approved in Europe as monotherapy for the treatment of partial seizures in adults with newly diagnosed epilepsy. This agent is also approved in Europe and USA as adjunctive therapy of focal seizures with or without secondary generalization with a proven safety and tolerability profile. ${ }^{13,14}$ A recent pooled analysis of clinical studies, where patients with epilepsy aged $\geq 65$ years treated with ZNS were enrolled, showed a favorable safety/tolerability profile. ${ }^{4}$ This, in combination with the negligible expected drug interactions, and the advantage of once-daily dosing, ${ }^{15}$ has led to an increasing
Correspondence: Andrea Romigi Neurophysiopathology Department, University of Rome Tor Vergata, Viale Oxford 8I, 00133 Rome, Italy

Tel +3906 20902107

$\mathrm{Fax}+390697655221$

Email a_romigi@inwind.it submit your manuscript | www.dovepress.com

Dovepress

http://dx.doi.org/10.2147/CIA.S50819 
interest in the potential use of this AED for epilepsy therapy in the elderly.

This review provides a comprehensive overview of available data on ZNS in the management of elderly people with epilepsy.

\section{Literature search strategy}

Studies discussed in this paper were searched by using the EMBASE and the PubMed databases from inception to November 28, 2014. The following keywords were used: "zonisamide", "epilepsy", and "elderly."

Further data were also searched from abstracts of International Conference Proceedings (Annual Meetings of the American Epilepsy Society and the Congresses of the International League Against Epilepsy). However, abstracts often do not show all relevant details and are not as rigorously peer reviewed as journal articles. Finally, the references of the selected papers were reviewed for additional articles.

\section{Pharmacology: mechanism of action}

ZNS has been documented to exhibit multiple mechanisms of action. ZNS has been shown to block both sodium and T-type $\mathrm{Ca}^{2+}$ channels. ${ }^{15}$ These mechanisms of action contribute to the stabilization of neuronal membranes and to the suppression of hypersynchronization.

In addition, ZNS has been reported to affect the glutamatergic and $\gamma$-aminobutyric acid-ergic neurotransmitter systems, ${ }^{16-20}$ to increase striatal and hippocampal concentration of dopamine and serotonin (total and extracellular) and to alter acetylcholine metabolism. Furthermore, ZNS has been demonstrated to weakly inhibit carbonic anhydrase activity. ${ }^{16,17}$ The clinical relevance of these mechanisms to antiseizure activity is still unclear. ${ }^{20}$

\section{Pharmacokinetic profile}

After oral administration, ZNS is rapidly absorbed and its oral bioavailability is virtually complete. The peak plasma concentration $\left(C_{\text {max }}\right)$ ranges between $2.3 \mathrm{mg} / \mathrm{mL}$ and $12.5 \mathrm{mg} / \mathrm{mL}$ (from $200 \mathrm{mg}$ to $800 \mathrm{mg}$ ), depending on the dosage, and is reached in 2.4-3.6 hours $\left(T_{\max }\right)$. The presence of food does not modify the extent of the drug absorption, but delays $T_{\text {max }}$ to $4-6$ hours. ${ }^{17}$

ZNS has an apparent volume of distribution of about $1.1-1.8 \mathrm{~L} / \mathrm{kg}$. Plasma ZNS levels increase proportionally for doses of $200-400 \mathrm{mg} .{ }^{21,22}$

ZNS is partially bound to the human serum albumin (about 50\%) and preferentially accumulated in erythrocytes. ${ }^{23}$ The elimination half-life of ZNS in plasma depends on the presence of cytochrome P450 (CYP)3A4 microsomal enzyme inducers (ie, phenobarbital, carbamazepine, and phenytoin) and ranges from about 50-70 hours in patients non-treated with enzyme inducers to $25-35$ hours in the treated ones. ${ }^{24}$ This long half-life allows the once-daily dosing. ${ }^{17}$ The apparent oral clearance is $15-20 \mathrm{~mL} / \mathrm{h} / \mathrm{kg}$ in patients non-treated concomitantly with enzyme inducers and $20-30 \mathrm{~mL} / \mathrm{h} / \mathrm{kg}$ in patients treated with enzyme inducers. ${ }^{24}$

The favorable pharmacokinetic profile supports the increasing use of ZNS. No major influences of ZNS on the pharmacokinetics of other concomitant medications have been reported. In particular, in patients on monotherapy with carbamazepine, ${ }^{25}$ valproic acid, ${ }^{26}$ phenytoin, ${ }^{27}$ or lamotrigine, ${ }^{28}$ the introduction of ZNS (up to $400 \mathrm{mg}$ /day) as add-on treatment did not affect the steady-state serum levels. In patients co-medicated with phenobarbital, carbamazepine, and phenytoin, the exposure to ZNS is decreased and the steady-state plasma levels are reached within a week. ${ }^{17}$

\section{Metabolism and excretion}

ZNS, before predominant excretion by renal route, undergoes hepatic biotransformation. ${ }^{29}$

In particular, ZNS is mainly metabolized in the open-ring metabolite, 2-sulfamoylacetyl phenol, by the CYP3A4 isoenzyme, in the $\mathrm{N}$-acetyl derivative by the $\mathrm{N}$-acetyltransferase, and, to a lesser extent, directly conjugated to the glucuronic acid. These metabolites are pharmacologically inactive. Of the excreted dose, $15 \%-30 \%$ was recovered as unchanged drug, $20 \%$ as ZNS glucuronide and $N$-acetyl ZNS, and $50 \%$ as the 2-sulfamoylacetyl phenol glucuronide..$^{29,30}$

\section{Pharmacokinetic profile in special groups}

The influence of aging on the pharmacokinetic profile of ZNS was assessed in a study ${ }^{16,31}$ conducted in healthy volunteers, 11 elderly (65-71 years) compared to 11 young (21-40 years), after oral administration of a single dose (300 mg). Wallace et $\mathrm{al}^{31}$ found that the elimination half-life of ZNS was 51.9 hours in the older subjects and 65.7 hours in the younger subjects, probably as a result of the smaller volume of distribution in the older population $(1.19 \mathrm{~L} / \mathrm{kg})$ compared with the non-elderly segment $(1.44 \mathrm{~L} / \mathrm{kg})$. No significant differences in renal clearance, mean plasma area under the serum concentration-time curve (AUC), and mean plasma clearance were found, and dose adjustments were not necessary. A single-dose study in subjects with renal impairment demonstrated that ZNS clearance was decreased 
significantly, and a 35\% AUC increase was observed in subjects with creatinine clearance values $<20 \mathrm{~mL} / \mathrm{min}^{16,23}$

\section{Efficacy}

There are limited data available on the efficacy of ZNS in the elderly population with focal epilepsy. Only one Phase IV randomized controlled trial and few uncontrolled studies reported data regarding the efficacy of ZNS in elderly patients with epilepsy.

\section{Randomized controlled study}

Results from a 16-week Phase IV randomized placebocontrolled study investigating the safety and tolerability profile of ZNS as add-on therapy in elderly patients with refractory focal epilepsy concomitantly treated with one or two AEDs ${ }^{32}$ (NCT01546688 clinicaltrial.gov/show/NCT01546688) show exploratory efficacy data on ZNS. This research has not yet been published but only a few data have been made available by the sponsor. Evidence from the results section of clinicaltrial.gov/show/NCT01546688 (last updated June 30, 2014) indicates that 33 patients (mean age 72.5 years) were enrolled in the treatment arm (five not completed study, none for lack of efficacy) and 18 (mean age 71.1 years) in the placebo arm (three not completed study, one for lack of efficacy). Although statistical analysis of efficacy data is not provided, the treatment group showed $75 \%$ of responders compared to $62 \%$ of responders in the placebo group during the last 28 days of the maintenance period. In addition, the median percent change in seizure frequency from baseline during the last 28 days of the maintenance period was similar in both the groups.

\section{Uncontrolled studies}

Data presented at the 25th International Epilepsy Congress Proceedings reported in a retrospective open-label study ${ }^{33}$ efficacy and safety of ZNS as monotherapy/add-on treatment in patients affected by focal and primary generalized epilepsy stratified by age. A large part of the elderly group ( $>60$ years) received concomitant antiepileptic treatment, and the mean dosage of ZNS was $475 \mathrm{mg} /$ day. Seizure freedom was achieved in $27 \%$ of elderly patients, while a $75 \%-99.9 \%$ reduction in seizure frequency was reached in $27 \%$. The authors did not state the exact number of older patients treated.

A further retrospective 8-week open-label study ${ }^{34}$ investigated 20 elderly patients (range 49-89 years, mean 64.1 years) with complex partial seizures treated with ZNS as add-on therapy. The ZNS dosage ranged from $100 \mathrm{mg}$ once daily to $100 \mathrm{mg}$ bid (twice daily) or $200 \mathrm{mg}$ once daily on the basis of clinical response. Three patients were affected by post-traumatic epilepsy, four by postanoxic damage, three were affected by stroke-related epilepsy, and ten by cryptogenic epilepsy. Three out of 20 patients $(15 \%)$ experienced seizure freedom, and 16 out of 20 patients (80\%) reported a reduction $>50 \%$ in seizure frequency. No dropouts were reported in the responder group. Tosches and Tisdell ${ }^{35}$ described 53 patients out of 60 patients identified ( $\geq 60$ years, mean 76 years, range 60-97) treated with ZNS for $\geq 3$ months. Thirty-seven patients were treated with ZNS as monotherapy (mean dose $200 \mathrm{mg} /$ day, mean duration 28.2 months). Twenty out of 37 patients $(54.1 \%)$ obtained seizure freedom, and a $\geq 50 \%$ reduction in seizure frequency was achieved in further five patients (13.5\%). The "add-on" group (16 patients, mean dose $268.8 \mathrm{mg} /$ day, mean duration 35.3 months) showed seizure freedom in five patients $(31.2 \%)$ and $\mathrm{a} \geq 50 \%$ reduction in seizure frequency in an additional seven patients (43.8\%). The authors also reported positive neurological effects with the improvement of extrapyramidal tremor $(n=2)$, improved awareness $(n=2), \operatorname{mood}(n=1)$, and also postherpetic neuralgia $(n=1)$ and restless legs syndrome $(n=1)$. In another retrospective study ${ }^{36}$ of 417 old adults from the Columbia AED database comparing effectiveness of ten AEDs, including ZNS in older adults ( $>55$ years), 30 patients with epilepsy (mean age 64 years) were taking ZNS (median dosage $300 \mathrm{mg}$ / day). The authors reported a 12-month seizure-freedom rate of $22.2 \%$ for ZNS $(n=18)$ similar to that obtained for the remaining AEDs except for lamotrigine and levetiracetam that showed seizure-freedom rates greater than $40 \%(54.1 \%, \mathrm{n}=85$ and $42.6 \%, \mathrm{n}=68$, respectively). The authors also compared 12-month retention rate (a combined evaluation of efficacy and tolerability) in 247 ( $\mathrm{n}=436$ subject-drug combinations) elderly patients newly treated with any AED over a period of 4 years. The highest retention rate was demonstrated for lamotrigine $(78.6 \%, \mathrm{n}=126)$, while $\mathrm{ZNS}$, valproate, and levetiracetam showed a $12-$ month retention rate of $68.2 \%(n=22)$, $69.6 \%(n=23)$, and $72.5 \%(n=102)$, respectively. The lowest retention rate was seen with oxcarbazepine $(23.5 \%, n=34)$. The subgroup evaluation of patients with refractory epilepsy ( $n=250$ subject-drug combinations) showed a retention rate of $90 \%$ for valproate $(\mathrm{n}=10)$, followed by lamotrigine $(77.8 \%$, $\mathrm{n}=63)$, levetiracetam $(71.8, \mathrm{n}=85)$, ZNS $(70 \%, \mathrm{n}=20)$, and topiramate $(66.7 \%, \mathrm{n}=15)$.

\section{Safety and tolerability Pooled analysis of data from clinical studies}

Recently, a pooled analysis of clinical data (five randomized, double-blind and two open-label clinical studies) specifically 
performed to evaluate safety and tolerability profile of ZNS in older epileptic patients ( $\geq 65$ years) compared to younger patients (18-65 years) has been published. ${ }^{4}$ In particular, Trinka et al analyzed data from 95 elderly patients and 1,389 younger adult patients and evaluated treatment-emergent adverse events (TEAEs). TEAEs were similar in both the groups ( $82 \%$ in elderly patients vs $84 \%$ in younger adult patients). In addition, the incidence of treatment-related TEAEs was less in older patients than in younger adults ( $56 \%$ vs $73 \%$, respectively). Although these findings may be related to different titration schedule and dosages, the authors did not report any data regarding titration for both the groups. The greater part of TEAEs was mild to moderate in each group. Severe TEAEs were reported less frequently in elderly subjects compared with the younger group (11.6\% vs $20.8 \%$ ). Dizziness (15\%), headache (13\%), somnolence $(12 \%)$, and fatigue (12\%), followed by nasopharyngitis ( $8 \%)$, constipation ( $7 \%$ ), diarrhea (6\%), confusion ( $6 \%$ ), pruritus $(6 \%)$, nausea (6\%), depression (5\%), insomnia (5\%), and loss of appetite (5\%) were the most frequently reported TEAEs in the elderly group compared with the younger group. Peripheral edema of mild to moderate intensity occurred in $4 \%$ of elderly subjects vs $1 \%$ in the non-elderly adults group.

The main treatment-related TEAEs in elderly patients were dizziness (14\%), sleepiness $(9 \%)$, fatigue $(8 \%)$, and pruritus $(6 \%)$. Only three serious treatment-related TEAEs (pancreatitis, rib fracture, and status epilepticus) were reported in elderly patients treated with ZNS as add-on therapy. One death was considered treatment related due to pancreatitis. TEAEs leading to ZNS withdrawal occurring in $\geq 2 \%$ of elderly patients were dizziness ( $4 \%$ ), headache $(2 \%)$, sleepiness $(2 \%)$, and confusion ( $2 \%)$. Trinka et al did not report significant changes in the clinical laboratory parameters and body weight in the elderly group. ${ }^{4}$

\section{Randomized controlled study}

Recently, a 16-week Phase IV randomized placebo-controlled study investigated the safety, tolerability, and efficacy of ZNS as adjunctive treatment in elderly patients affected by refractory focal epilepsy concomitantly treated with one or two AEDs (NCT01546688 clinicaltrial.gov/show/NCT01546688, last updated June 30, 2014). The clinical data of 33 treated patients 18 patients in the placebo arm were reported. Serious adverse events were $6 / 33$ (18.2\%) with ZNS versus 1/18 (5.6\%) with placebo, whereas other adverse events were found in 17 out of $33(51.5 \%)$ elderly patients with epilepsy and in eight out of 18 (44.4\%) patients in the placebo group. Serious adverse events reported in treated group were cardiovascular insufficiency, pancreatitis, sudden death, rib fracture, convulsion, status epilepticus, vertebrobasilar insufficiency, and renal failure (1/33, 3.03\% each).

Regarding the other adverse events, the most reported in the ZNS group were dizziness $(5 / 33,15.15 \%$ vs $2 / 18,11.11 \%$ in the placebo group), somnolence $(5 / 33,15.15 \%$ vs $0 / 18$, $0 \%$ in the placebo group), and headache $(4 / 33,12.12 \%$ vs $0 / 18,0 \%$ in the placebo group).

\section{Uncontrolled studies}

In a retrospective 8 -week open-label study ${ }^{34}$ in 20 elderly patients (mean 64 years, range 49-89) with complex partial seizures under ZNS as add-on therapy, three patients discontinued ZNS (one for skin rash, one for visual hallucinations, and one for drowsiness). In addition, among 16 responder patients, five reported mild gastrointestinal upset and sleepiness without leading to ZNS discontinuation.

In a further retrospective open-label study ${ }^{35}$ conducted in 60 elderly epileptic patients treated with ZNS (as monotherapy or add-on treatment), six patients $(10 \%)$ discontinued ZNS, five due to unspecified adverse events (8.3\%). The most frequent TEAEs were gastrointestinal upset, skin rash, and mild somnolence $(2 / 60,3.3 \%$ each) followed by anorexia, vision changes, headache, and lightheadedness $(1 / 60,1.7 \%$ each). In a retrospective analysis ${ }^{36}$ comparing effectiveness of ten AEDs in old adults, the most frequent TEAEs leading to treatment discontinuation and/or to the dose change in more than $2 \%$ of patients in the ZNS-treated patients $(n=30)$ were as follows: cognitive and gastrointestinal (10\%-19.9\%); allergies, dizziness, and headache (5\%-9.9\%); drowsiness, psychiatric effects, sleep difficulty, and vision (2\%-4.9\%).

\section{Discussion}

Seizures and epilepsy represent the third most frequent neurological disorder in the elderly. The incidence of epilepsy increases exponentially in people aged $\geq 65$ years. The treatment of epilepsy in elderly patients can be challenging due to the fact that toxicity may be associated with concomitant treatments or medical conditions such as stroke, Alzheimer's, or Parkinson's disease. Several aspects may become complex treatment choices in elderly patients with epilepsy; therefore, physicians should pay attention in the management of seizures and epilepsy in this age-specific group. AEDs choice and dosing should consider the age-related physiological changes, comorbidities, concomitant treatment, and drug interactions. Although the primary goals of epilepsy therapy are similar in all ages, the abovementioned concerns become noteworthy issues managing epilepsy in older people. Thus, 
drug selection needs to take into account not only efficacy but also tolerability and safety issues. Desirable characteristics of an AED for the management of epilepsy in aging are as follows: i) favorable safety profile; ii) absence of drug-drug interactions (concomitant treatment with AEDs and other medications); iii) weak influence on cognitive function; and iv) long half-life in order to allow one or two daily intakes. ${ }^{38}$ Older patients with new onset epilepsy are often started on first-generation AEDs despite their poor tolerability. However, the second-generation AEDs are characterized by a better pharmacokinetic and tolerability profile and are best suited for the management of epilepsy in the elderly. ${ }^{24}$ The pharmacokinetic profile of ZNS (long half-life, mild drug-drug interactions, and no induction or inhibition of the hepatic microsomal enzymes) can be considered favorable for the management of epilepsy in the elderly. ${ }^{15,29}$ This review showed several concerns. First, to offer the most recent estimation of the topic, we embraced abstracts from congress proceedings. Often, abstracts may not include significant aspects and are not subject to peer-reviewing processes. In addition, we derived data from the abstract itself and not from the posters. Therefore, some additional data regarding safety and efficacy of ZNS in elderly patients with epilepsy may have been missed by this approach. In addition, we cannot obtain comparisons regarding the frequency of adverse events between add-on and monotherapy studies. Although the few data obtained from the literature may be encouraging, we are far from obtaining generalizations regarding ZNS as AED in elderly population. Efficacy data from a single controlled study on efficacy of ZNS in elderly patients ${ }^{32}$ do not allow any conclusion because the data analysis is ongoing, and the sample size seems too small. ZNS is often well tolerated, but some authors suggested that its use may be limited by somnolence, psychiatric, and cognitive adverse effects. ${ }^{37,39}$ Psychiatric adverse events include depression, irritability, aggressive behavior, and psychosis, whereas cognitive adverse events comprise language impairment, cognitive slowing, memory impairment, and reduced mental concentration in a manner similar to topiramate. ${ }^{39,40}$ Although specific findings regarding psychiatric and cognitive profile of ZNS in older patients are lacking, cognitive and psychiatric adverse effects may be common during ZNS treatment, but they are related to specific risk factors (ie, past psychiatric history, symptomatic generalized epilepsy, number of concomitant AEDs, and ZNS serum levels) ${ }^{39}$ with a low discontinuation rate in selected patients. ${ }^{41}$ The favorable ZNS pharmacokinetic profile, the lack of interaction, may represent the strength of the choice of ZNS in the elderly.
Although efficacy data are lacking, safety and tolerability of ZNS were recently evaluated in a pooled data analysis of five double-blind and two open-label studies conducted by Trinka et $\mathrm{al}^{4}$ in patients aged $\geq 65$ years. In this analysis as well as in other uncontrolled studies, it has been reported that elderly patients treated with ZNS did not present more adverse events than the non-elderly. However, the relatively high frequency of cognitive and sedative adverse effects (ie, somnolence, headache, and dizziness) should be taken into account when ZNS is used in elderly patients. ${ }^{13,37}$ On the other hand, ZNS is effective in several disorders (ie, migraine, Parkinson's disease, obesity, and sleep disorders) that may affect elderly subjects. ${ }^{14,42-44}$ Therefore, ZNS may represent a useful option for the obese patients due to weight loss, for migraine, for patients affected by Parkinson's disease, and for sleep complaints. Recently, Eskandari et $\mathrm{al}^{42}$ showed a significant decrease of sleep apnea in a randomized placebocontrolled design independent of weight loss and probably due to carbonic anhydrase inhibition. On the contrary, treatment with ZNS should be avoided in patients with multiple or severe prior allergy/rash and renal stones. In a survey of experts for the treatment of symptomatic focal epilepsy in the elderly conducted in 2005, ZNS was recognized as first-line treatment by $39 \%$ and as second line by $56 \% .{ }^{45}$ Lamotrigine, levetiracetam, gabapentin, oxcarbazepine, and carbamazepine were considered as first-line AEDs by $98 \%, 95 \%$, $71 \%, 61 \%$, and $59 \%$. $^{45}$

\section{Conclusion}

Based on our review, we conclude that, to date, there is no satisfactory evidence on the efficacy and tolerability of ZNS in the treatment of elderly patients with epilepsy, although the data from a limited number of studies are encouraging. Large controlled prospective studies are needed to establish the real role of newer generation AEDs, such as ZNS, in the management of epilepsy in the elderly.

\section{Disclosure}

$\mathrm{VF}$ is a former employee of Eisai s.r.l., Italy. AR, GDG, EAF, $\mathrm{CF}$, and GV have no conflicts of interest to declare.

\section{References}

1. Johnston A, Smith PE. Epilepsy in the elderly. Expert Rev Neurother. 2010;10:1899-1910.

2. Stephen LJ, Brodie MJ. Epilepsy in elderly people. Lancet. 2000;355: 1441-1446.

3. Faught E, Richman J, Martin R, et al. Incidence and prevalence of epilepsy among older U.S. Medicare beneficiaries. Neurology. 2012;78:448-453.

4. Trinka E, Giorgi L, Patten A, Segieth J. Safety and tolerability of zonisamide in elderly patients with epilepsy. Acta Neurol Scand. 2013;128:422-428. 
5. Trinka E. Epilepsy: comorbidity in the elderly. Acta Neurol Scand Suppl. 2003;180:33-36.

6. Brodie MJ, Elder AT, Kwan P. Epilepsy in later life. Lancet Neurol. 2009;8:1019-1030.

7. Saetre E, Perucca E, Isojärvi J, Gjerstad L; LAM 40089 Study Group. An international multicenter randomized double-blind controlled trial of lamotrigine and sustained-release carbamazepine in the treatment of newly diagnosed epilepsy in the elderly. Epilepsia. 2007;48: 1292-1302.

8. Rowan AJ, Ramsay RE, Collins JF, et al; VA Cooperative Study 428 Group. New onset geriatric epilepsy: a randomized study of gabapentin, lamotrigine, and carbamazepine. Neurology. 2005;64:1868-1873.

9. Brodie MJ, Overstall PW, Giorgi L. Multicentre, double-blind, randomised comparison between lamotrigine and carbamazepine in elderly patients with newly diagnosed epilepsy. The UK Lamotrigine Elderly Study Group. Epilepsy Res. 1999;37:81-87.

10. Werhahn KJ, Klimpe S, Balkaya S, Trinka E, Krämer G. The safety and efficacy of add-on levetiracetam in elderly patients with focal epilepsy: a one-year observational study. Seizure. 2011;20:305-311.

11. Kutluay E, McCague K, D’Souza J, Beydoun A. Safety and tolerability of oxcarbazepine in elderly patients with epilepsy. Epilepsy Behav. 2003; 4:175-180.

12. Giorgi L, Gomez G, O’Neill F, Hammer AE, Risner M. The tolerability of lamotrigine in elderly patients with epilepsy. Drugs Aging. 2001;18:621-630.

13. Zaccara G, Specchio LM. Long-term safety and effectiveness of zonisamide in the treatment of epilepsy: a review of the literature. Neuropsychiatr Dis Treat. 2009;5:249-259.

14. Romigi A, Izzi F, Placidi F, et al. Effects of zonisamide as add-on therapy on sleep-wake cycle in focal epilepsy: a polysomnographic study. Epilepsy Behav. 2013;26:170-174.

15. Brodie MJ, Ben-Menachem E, Chouette I, Giorgi L. Zonisamide: its pharmacology, efficacy and safety in clinical trials. Acta Neurol Scand Suppl. 2012;194:19-28.

16. Shah J, Shellenberger K, Canafax D. ZNS: chemistry, biotransformation, and pharmacokinetics. In: Levy R, Mattson R, Meldrum B, editors. Antiepileptic Drugs. 5th ed. Philadelphia (PA): Lippincott Williams and Wilkins; 2002:872-884.

17. Eisai Pharmaceuticals Inc. Zonegran (Prescribing Information). Teaneck (NJ): Eisai Pharmaceuticals Inc; 2004.

18. Wroe SJ. Zonisamide. In: Simon Shorvon EP, Engel J, editors. Treatment of Epilepsy. 3rd ed. Oxford: Wiley-Blackwell; 2009:1200.

19. Ueda Y, Doi T, Tokumaru J, Willmore LJ. Effect of zonisamide on molecular regulation of glutamate and GABA transporter proteins during epileptogenesis in rats with hippocampal seizures. Brain Res Mol Brain Res. 2003;116:1-6.

20. Okada M, Kaneko S, Hirano T, et al. Effects of zonisamide on dopaminergic system. Epilepsy Res. 1995;22:193-205.

21. Peters DH, Sorkin EM. Zonisamide. A review of its pharmacodynamic and pharmacokinetic properties, and therapeutic potential in epilepsy. Drugs. 1993;45:760-787.

22. Faught E, Ayala R, Montouris GG, Leppik IE; Zonisamide 922 Trial Group. Randomized controlled trial of zonisamide for the treatment of refractory partial-onset seizures. Neurology. 2001;57: 1774-1779.

23. Masuda Y, Noguchi H, Karasawa T. Evidence against a significant implication of carbonic anhydrase inhibitory activity of zonisamide in its anticonvulsive effects. Arzneimittelforschung. 1994;44:267-269.

24. Italiano D, Perucca E. Clinical pharmacokinetics of new-generation antiepileptic drugs at the extremes of age: an update. Clin Pharmacokinet. 2013;52:627-645.

25. Ragueneau-Majlessi I, Levy RH, Bergen D, et al. Carbamazepine pharmacokinetics are not affected by zonisamide: in vitro mechanistic study and in vivo clinical study in epileptic patients. Epilepsy Res. 2004; 62:1-11.
26. Ragueneau-Majlessi I, Levy RH, Brodie M, Smith D, Shah J, Grundy JS. Lack of pharmacokinetic interactions between steady-state zonisamide and valproic acid in patients with epilepsy. Clin Pharmacokinet. 2005;44:517-523.

27. Levy RH, Ragueneau-Majlessi I, Garnett WR, et al. Lack of a clinically significant effect of zonisamide on phenytoin steady-state pharmacokinetics in patients with epilepsy. J Clin Pharmacol. 2004;44: 1230-1234.

28. Levy RH, Ragueneau-Majlessi I, Brodie MJ, Smith DF, Shah J, Pan WJ. Lack of clinically significant pharmacokinetic interactions between zonisamide and lamotrigine at steady state in patients with epilepsy. Ther Drug Monit. 2005;27:193-198.

29. Sills G, Brodie M. Pharmacokinetics and drug interactions with zonisamide. Epilepsia. 2007;48:435-441.

30. Frampton JE, Scott LJ. Zonisamide: a review of its use in the management of partial seizures in epilepsy. CNS Drugs. 2005;19:347-367.

31. Wallace J, Shellenberger K, Groves L. Pharmacokinetics of zonisamide in young and elderly subjects [abstract no.6.049]. Epilepsia. 1998;39:190-191.

32. Eisai Limited. A study to evaluate the safety and tolerability and explore the efficacy of zonisamide as add-on therapy in elderly patients with refractory partial seizures; 2014. Available from: http:// www.clinicaltrials.gov/show/show/NCT01546688. NLM identifier: NCT01546688. Accessed November 20, 2014.

33. Ramsay RE. Zonisamide (ZNS, Zonegran (R)): efficacy and safety stratified by patient age, p292. abstracts from the 25th International Epilepsy Congress Lisbon, Portugal October 12-16, 2003. Epilepsia. 2003; 44:1-223.

34. Hasegawa H. Zonisamide adjunct therapy for elderly intractable seizure in VA outpatient clinic. In: American Epilepsy Society Annual Meeting, 2003; Abstract 1.038. Available from: www.aesnet.org/ meetings_events/annual_meeting_abstracts/view/2084

35. Tosches W, Tisdell J. Epilepsy in the older patient: US experience with long-term zonisamide therapy (p535). 7th European Congress on Epileptology Helsinki, Finland, 2-6 July 2006. Epilepsia. 2006;47:117-148.

36. Arif H, Buchsbaum R, Pierro J, et al. Comparative effectiveness of 10 antiepileptic drugs in older adults with epilepsy. Arch Neurol. 2010; 67:408-415.

37. Arif H, Mendiratta A, Hirsch L. Management of epilepsy in the elderly. In: Shorvon S, Perucca E, Engel J Jr, editors. The Treatment of Epilepsy. Hoboken: Wiley-Blackwell; 2009:203-218.

38. Bergey G. Initial treatment of epilepsy: special issues in treating the elderly. Neurology. 2004;63:S40-S48.

39. White JR, Walczak TS, Marino SE, Beniak TE, Leppik IE, Birnbaum AK. Zonisamide discontinuation due to psychiatric and cognitive adverse events: a case-control study. Neurology. 2010;75:513-518.

40. Romigi A, Cervellino A, Marciani MG, et al. Cognitive and psychiatric effects of topiramate monotherapy in migraine treatment: an open study. Eur J Neurol. 2008;15:190-195.

41. Cavanna AE, Seri S. Psychiatric adverse effects of zonisamide in patients with epilepsy and mental disorder comorbidities. Epilepsy Behav. 2013;29:281-284.

42. Eskandari D, Zou D, Karimi M, Stenlöf K, Grote L, Hedner J. Zonisamide reduces obstructive sleep apnoea: a randomised placebocontrolled study. Eur Respir J. 2014;44:140-149.

43. Gadde KM, Kopping MF, Wagner HR 2nd, Yonish GM, Allison DB, Bray GA. Zonisamide for weight reduction in obese adults: a 1-year randomized controlled trial. Arch Intern Med. 2012;172:1557-1564.

44. Mohammadianinejad SE, Abbasi V, Sajedi SA, et al. Zonisamide versus topiramate in migraine prophylaxis: a double-blind randomized clinical trial. Clin Neuropharmacol. 2011;34:174-177.

45. Karceski S, Morrell MJ, Carpenter D. Treatment of epilepsy in adults: expert opinion, 2005. Epilepsy Behav. 2005; 7(Supp1 1):S1-S64; quiz S65-S67. 
Clinical Interventions in Aging

\section{Publish your work in this journal}

Clinical Interventions in Aging is an international, peer-reviewed journal focusing on evidence-based reports on the value or lack thereof of treatments intended to prevent or delay the onset of maladaptive correlates of aging in human beings. This journal is indexed on PubMed Central, MedLine,

CAS, Scopus and the Elsevier Bibliographic databases. The manuscript management system is completely online and includes a very quick and fair peer-review system, which is all easy to use. Visit http://www.dovepress. $\mathrm{com} /$ testimonials.php to read real quotes from published authors.

Submit your manuscript here: http://www.dovepress.com/clinical-interventions-in-aging-journal 